Ann. Zootech., r965, 14 (4), 409-4I5.

\title{
EFFET DU NOMBRE DE SPERMATOZOÏDES MOBILES UTILISÉS PAR INSÉMINATION, SUR LE POUVOIR FÉCONDANT DU SPERME CONGELÉ DE TAUREAUX.
}

\author{
M. GOFFAUX \\ avec la collaboration technique de J. C. Tourneur, Union nationale des Coopératives \\ d'Élevage d'Insémination artificiele \\ Laboratoire de la Physiologie de la Reprodiction, \\ Centre national de Recherches zootechniques, Jouy-en-Josas (Seine-et-Oise)
}

\section{SOMMAIRE}

Seize éjaculats provenant de 9 taureaux Charolais ont été dilués dans un milien lait écréméglycérol et congelés selon un procédé classique au moyen d'un bain d'alcool refroidi à l'aide de glace carbonique. Chaque éjaculat a été divisé en 3 fractions d'égal volume contenant respectivement 24, $3^{\text {r }}$ et 40 millions de spermatozoïdes mobiles par millilitre avant congélation, nombres réduits à i 1,0 i 4,2 et 18,3 millions après congélation.

Huit cent soixante-dix doses (ampoules) en moyenne ont été employées pour chacun des traitements. Les pourcentages de non-retours à $90-120$ jours ont été respectivement de $6 \mathrm{r}, 6--62,9-$ 66,5 . Seule la différence entre les résultats obtenus avec ir,o et 18,3 millions de spermatozoïdes mobiles après congélation est significative $(\mathrm{P}<0,05)$. La tendance indiquée montre que l'emploi du sperme congelé en insémination bovine requiert la présence, au moment de l'intervention, d'un nombre de spermatozoïdes mobiles par dose sensiblement supérieur à la valeur minimum recommandée lorsque le sperme est utilisé à l'état frais.

\section{INTRODUCTION}

Il est connu depuis longtemps qu'on peut réduire le nombre total de spermatozoïdes de taureau utilisés à l'état frais à moins de 20 millions par insémination (SAlisbury, I96I) et récemment FooTe et Dunv, (I962) ont obtenu des pourcentages de non-retours également élevés avec des doses de semence conservées pendant 3 jours et contenant 5 ou ro millions de spermatozoïdes mobiles au conditionnement.

On peut se demander s'il est possible de généraliser ces conclusions à l'utilisation du sperme congelé, compte tenu du seul nombre de spermatozoïdes mobiles 
par dose au moment de l'insémination, puisque ni la longévité, ni l'activité métabolique du spermatozoïde mobile après congélation ne sont équivalentes à celles de son homologue non congelé (O'DEL, et ALmQUist, I958 ; Sul.Livan et MiXNER, I963). ERICKSON et GRAHAM (I959) constatent que des doses de semence renfermant 30 millions de spermatozoïdes mobiles avant congélation montrent un pouvoir fécondant significativement supérieur à celles qui n'en contiennent que ro millions. Cependant, EMmens et Mar'Tin (I96I) n'observent aucune différence dans le pouvoir fécondant de doses apportant $13,3-20$ ou 30 millions de spermatozoïdes totaux par insémination.

L'expérimentation rapportée ici a été réalisée pour éprouver l'effet de 3 concentrations de spermatozoïdes mobiles avant congélation, voisines du maximum (30 millions) cité dans la bibliographie.

\section{MATÉRIEI, ET MÉTHODES}

Le dilueur employé est le même que celui décrit précédemment (Goffaux, I964). La composition finale comprend, pour roo $\mathrm{ml}$ : lait (écrémé reconstitué contenant ro $\mathrm{g}$ de matière sèche pour $90 \mathrm{~g}$ d'eau), $90 \mathrm{ml}$; glycérol, $10 \mathrm{ml}$; fructose, $\mathrm{I}, 25 \mathrm{~g}$; pénicilline, $45.000 \mathrm{UI}$; dihydrostreptomycine $45 \mathrm{ing}$.

Apprès sélection, i6 éjaculats provenant de 9 taureaux de race Charnlaise ont été utilisés, chacun résultant le plus souvent du mélange de 2 échantillons de semence. Seuls ont été retenus pour la congélation les éjaculats montrant un pourcentage de spermatozoïdes mobiles après glycérolisation égal ou supérieur à $50 \mathrm{p}$. 100 et apportant au minimum 6 milliards de sperinatozoïdes totaux. Les éjaculats montrant moins de 20 p. roo de spermatozoïdes mobiles aprés congélation ont été éliminés ainsi que ceux pour lesquels plus des $2 / 3$ des spermatozoïdes ont été inactivés au cours de l'opération.

Chaque éjaculat utilisé a été partagé, I heure 30 après glycérolisation, en 3 fractions d'égal volume contenant respectivement $24,3 \mathrm{I}$ et 40 millions de spərmatozoïdes mobiles par millilitre, et conditionné en ampoules par doses de I millilitre. Le temps d'équilibration a été fixé à 4 heures. Ies congélations ont été réalisées au moyen d'un bain d'alcool refroidi à l'aide de glace carbonique à raison de $1^{\circ} \mathrm{C} / \mathrm{mn}$ entre $+4^{\circ} \mathrm{C}$ et $-I^{\circ} \mathrm{C}$ et de $5^{\circ} \mathrm{C} / \mathrm{mn}$ entre $-15^{\circ} \mathrm{C}$ et $-70^{\circ} \mathrm{C}$.

Les doses ont été conservées à - $196^{\circ} \mathrm{C}$ pendant 3 à 8 mois. Quatre inséminateurs, préalablement entrânés à la manipulation de sperme congelé, ont reçu, à 6 ampoules près, le même nombre de doses à inséminer pour chaque récolte et chaque traitement. Pour simplifier leur travail, l'ensemble de la semence congelée a été divisée en 3 lots utilisés successivement, chaque lot correspondant aux doses relatives à 3 taureaux. La tenue à jour d'un état de la semence en stock a permis l'utilisation quasi simultanée des doses des 3 traitements pour chaque taureau, évitant toute erreur dûe à d'éventuelles interactions entre traitement et période d'utilisation ou entre traitement, taureau et période d'utilisation.

\section{RÉSULTATS}

La qualité du sperme inséminé est présentée dans le tableau I et le tableau 2 indique les données relatives au pouvoir fécondant de la semence pour chacune des 3 concentrations de spermatozoïdes mobiles étudiées.

Malgré la sélection exercée avant et après congélation (sur 50 échantillons de semence récoltés, 35 ont été congelés et 30 ont été finalement retenus en vue de l'insémination) les éjaculats utilisés montrent une chute du pourcentage de spermatozoïdes mobiles supérieure à celle habituellement constatée au cours del'opération. 


\section{TABLEAU I}

Caractères des éjaculats utilisés

\begin{tabular}{|c|c|c|c|}
\hline Caractère & $\begin{array}{l}\text { Valeurs } \\
\text { extrêmes }\end{array}$ & Moyennes & $\begin{array}{l}\text { licart } \\
\text { type }\end{array}$ \\
\hline $\begin{array}{l}\text { Pourcentage de spz. mobiles avant congéla- } \\
\quad \text { tion }(16 \text { éjaculats) } \ldots \ldots \ldots \ldots \ldots \ldots \ldots \ldots\end{array}$ & $50-70$ & 61,6 & 6,1 \\
\hline $\begin{array}{l}\text { Pourcentage de spz. mobiles après congéla- } \\
\text { tion }(16 \text { éjaculats) } \ldots \ldots \ldots \ldots \ldots \ldots \ldots \ldots\end{array}$ & $22-35$ & 28,1 & 4,7 \\
\hline $\begin{array}{c}\text { Nombre évalué de spz. mobiles par ml } \\
\text { appès congélation }\left(10^{6}\right)\end{array}$ & & & \\
\hline $\begin{array}{l}24.10^{6} \mathrm{spz} \text {. mobiles par } \mathrm{ml} \text { avant congélation.. } \\
31.10^{6} \mathrm{spz} \text {. mobiles par ml avant congélation. }\end{array}$ & $\begin{array}{r}8-14 \\
10-18\end{array}$ & $\begin{array}{l}11,0 \\
14,2\end{array}$ & $\begin{array}{l}2,0 \\
2,8\end{array}$ \\
\hline $40.10^{6} \mathrm{spz}$. mobiles par ml avant congélation. . & $13-23$ & 18,3 & $3,{ }^{\prime}$ \\
\hline $\begin{array}{l}\text { Pourcentage de non-retours à } 90-120 \text { jours, } \\
\text { par éjaculat } \ldots \ldots \ldots \ldots \ldots \ldots \ldots \ldots \ldots \ldots\end{array}$ & $45-73$ & 63,4 & 7,1 \\
\hline
\end{tabular}

TABLEAU 2

Pourcentage de non-retours (90-120 jours) par traitements et taureaux

\begin{tabular}{|c|c|c|c|c|c|c|c|c|c|}
\hline \multirow{3}{*}{ Taureaux } & \multicolumn{6}{|c|}{$\begin{array}{l}\text { Nombre de spermatozoïdes mobiles } \\
\text { par dose avant congélation }\end{array}$} & \multirow{2}{*}{\multicolumn{2}{|c|}{ Moyenne }} & \\
\hline & \multicolumn{2}{|c|}{$2 / 4$ millions } & \multicolumn{2}{|c|}{31 millions } & \multicolumn{2}{|c|}{40 millions } & & & $\chi^{2}$ \\
\hline & $\begin{array}{c}\text { nombre } \\
\text { insémin. } \\
1^{\text {res }}\end{array}$ & $\begin{array}{l}\% \text { non } \\
\text { retours }\end{array}$ & $\begin{array}{c}\text { nombre } \\
\text { insémin. } \\
1^{\text {res }}\end{array}$ & $\begin{array}{l}\% \text { non } \\
\text { retours }\end{array}$ & $\begin{array}{c}\text { nombre } \\
\text { insémin. } \\
1^{\text {rea }}\end{array}$ & $\begin{array}{l}\% \text { non } \\
\text { retours }\end{array}$ & $\begin{array}{c}\text { nombre } \\
\text { insémin. } \\
1^{\text {res }}\end{array}$ & $\begin{array}{l}\% \text { non } \\
\text { retours }\end{array}$ & taureilı \\
\hline 019 & 76 & 56,6 & 76 & 69,7 & 78 & 61,5 & 230 & 62,6 & 2,87 \\
\hline 037 & 65 & 50,8 & 62 & 56,5 & 71 & 69,0 & 198 & 59,1 & $4.99_{1}^{\prime}$ \\
\hline 059 & 128 & 64,1 & 125 & 61,6 & 120 & 70,0 & 373 & 65,1 & 2,60 \\
\hline 062 & 125 & 68,8 & 132 & 65,2 & 131 & 67,2 & 388 & 67,0 & 0,39 \\
\hline 034 & 154 & 68,8 & 149 & 70,5 & 155 & 70,3 & 458 & 69,9 & 0,11 \\
\hline 048 & 148 & 58,1 & 134 & $6 /, 9$ & 140 & 71,2 & 428 & 64,7 & 5,55 \\
\hline 033 & 30 & 63,3 & 29 & 55,2 & 40 & 62,5 & 99 & 60,6 & $0,5=$ \\
\hline 113 & 90 & 54,4 & 91 & 49,5 & 91 & 46,2 & 272 & 50,0 & 1,26 \\
\hline 002 & 50 & 58,0 & 50 & 58,0 & 56 & 73,2 & 156 & 63,5 & 3,58 \\
\hline Total & 866 & 61,6 & 848 & 62,9 & 888 & 66,5 & 2602 & 63,6 & 21,8: \\
\hline
\end{tabular}

Le pourcentage de non-retours à 90-I 20 jours varie très significativement selon les taureaux $\left(\chi^{2}=25,7\right.$ pour $\left.8 \mathrm{DL}\right)$ et s'élève lorsqu'on augmente le nombre de spermatozoïdes mobiles par dose avant congélation : $6 \mathrm{I}, 6$ p. Ioo pour 24 millions 62,9 p. Ioo pour 31 millions, 66,5 p. Ioo pour 40 millions.

Cependant, d'après la valeur trouvée pour la somme des $\chi^{2}$ particuliers à chaque taureau, la classification des inséminations selon les 3 traitements ne parait pas 
contredire 1'hypothèse de l'homogénéité du pouvoir fécondant des doses utilisées (tabl. $3_{1}$ ). Ein outre, la valeur de $\chi^{2}$ calculée sur les totaux montre que les pourcentages de non-retours relatifs aux inséminations correspondant aux 3 traitements ne diffèrent pas significativement. L'hétérogénéité normale indique que les doses de semence paraissent avoir été employées dans des conditions satisfaisantes.

\section{TABLEAU 3}

Interprétation de l'effet des traitements

Io Toutes données. Épreuve par taureau. F́preuve sur les totaux. Test de la constance des déviations.

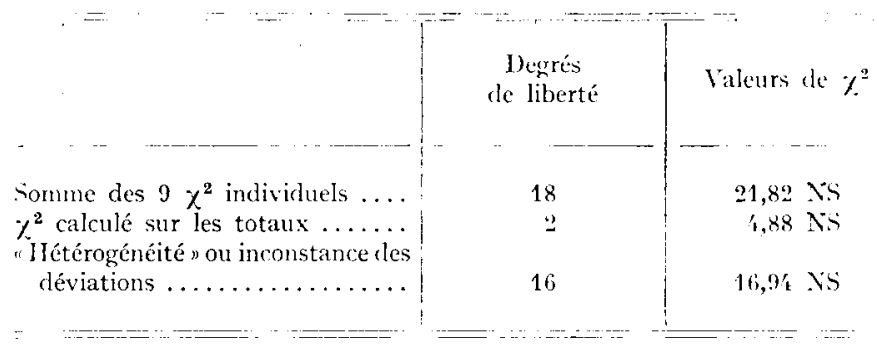

$2^{0}$ Données limitées aux traitements extrêmes $\left(24 \cdot \mathrm{IO}^{6}\right.$ et $40 . \mathrm{rO}^{6}$ spermatozoïdes mobiles avant congélation).

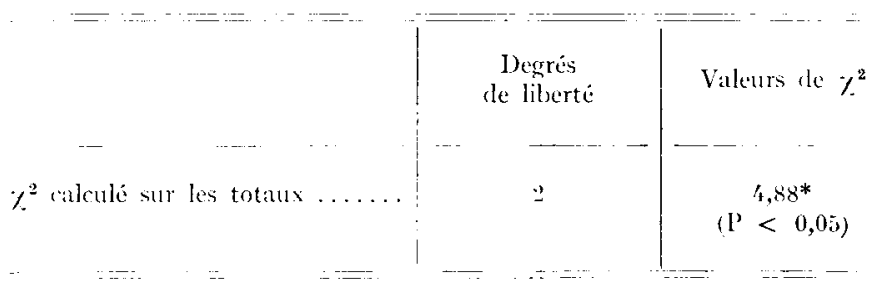

$3^{0}$ 'Toutes données. Pourcentage de non-retour par traitement après répartition des éjaculats en 2 classes selon le pourcentage de réanimation et $\chi^{2}$ correspondants pour 2 degrés de liberté. (Le nombre d'inséminations est indiqué entre parenthèses).

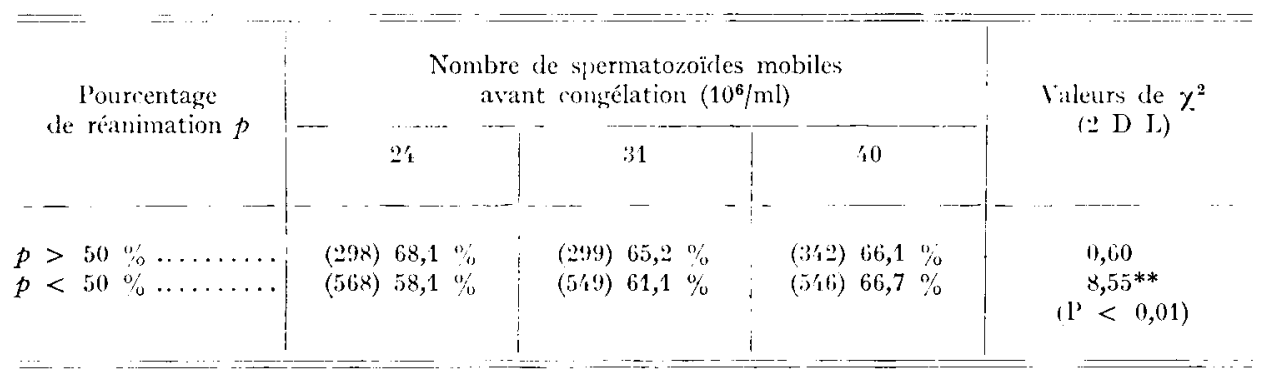

Toutefois, lorsqu'on ne considère que les inséminations réalisées à partir des doses contenant 24 ou 40 millions de spermatozoïdes mobiles avant congélation 
(tabl. 32 ), la nouvelle valeur de $\chi^{2}$ calculée sur les totaux montre que les 2 traitements opposés ont des effets significativement différents $(\mathrm{P}<0,05)$.

La répartition des éjaculats en 3 classes, d'après le pourcentage moyen de nonretours pour chacun d'eux, ne permet pas d'affirmer que les traitements ont un effet plus marqué sur les éjaculats montrant un pouvoir fécondant inférieur à une certaine limite.

En revanche, malgré le petit nombre d'inséminations par groupe, la classification des éjaculats selon le pourcentage de réanimation (1) parait indiquer que l'effet đu nombre de spermatozoïdes mobiles après congélation n'est sensible qu'au-dessous d'un plafond situé entre io et I.5 millions (tabl. $3_{3}$ ).

\section{CONCLESION}

Les 3 nombres de spermatozoïdes mobiles avant congélation comparés dans cet essai ont été choisis de telle sorte que l'un d'eux soit nettement supérieur au nombre de 30 millions par millilitre, maximum éprouvé dans l'expérimentation d'ERICKSON et GRAHAM (I959) et nombre généralement considéré comme optimum. Toutefois, en raison de la chute anormalement importante du pourcentage de spermatozoïdes mobiles au cours des congélations réalisées dans l'expérimentation présentée ici, les 3 traitements essayés se retrouvent exactement dans la zône étudiée par ces auteurs lorsqu'on les distingue par le nombre de spermatozoïles mobiles par millilitre après congélation. Afin d'éviter toute ambiguïté dans l'énoncé des résultats, ce dernier caractère semble donc devoir être retenu de préférence au nombre total de spermatozoïles ou au nombre de spermatozoïdes mobiles par millilitre de semence avant congélation.

La grande dispersion du nombre de spermatozoïlles mobiles après congélation dans les doses relatives à un traitement donné et le large empiètement réciproque des 3 populations d'insémination qui en résulte (tabl. r) paraissent avoir eu 2 conséquences opposées contrariant la détermination des effets des traitements :

I'une part le chevauchement des populations d'inséminations est capable d'expliquer l'absence de différence significative entre les 3 traitements considérés dans leur ensemble.

Mais d'autre part, cette dispersion s'est traduite par l'abaissement du nombre de spermatozoïdes mobiles après congélation au-dessous de $\mathrm{I} 0$ millions pour le tiers des doses du traitement le moins favorable; l'examen du tableau 3 suggère que ce seul fait a pu entraîner la constatation d'une différence significative entre les 2 traitements extrêmes.

I,e mode de classification des éjaculats selon le pourcentage de réanimation semble reposer sur un critère ambigu, à la fois qualitatif (aptitude à subir la congélation) et quantitatif (nombre de spermatozoïdes mobiles après congélation).

Toutefois, lorsque le nombre de spermatozoïdes mobiles par dose dépasse ro à I5 millions après congélation, l'examen du tableau $3_{3}$ montre que le pouvoir

(1) Pourcentage de réanimation : Kapport du nombre de spermatozoïdes mobiles après congélation au nombre de spennatozodes m.biles avant congélation. 
fécondant est assez peu lié au pourcentage de réanimation et que l'aspect quantitatif du critère est déterminant dans cette expérimentation.

L'ensemble de ces résultats paraît confirmer ceux d'ERIckson et Graham montrant une différence presque significative entre le pouvoir fécondant des doses conte nant 7 ou I4 millions mais pas de différence entre les doses contenant I4 ou 2 I millions de spermatozoïdes mobiles après congélation.

Il demeure toutefois que le nombre minimum de spermatozoïdes par dose de sperme congelé se situe assez nettement au-dessus du nombre de 5 millions indiqué par Foote et DUNN (I962) pour le sperme frais dilué en C. U. E. Ces résultats, s'ils se confirment, montrent donc qu'il est nécessaire de compenser par l'apport d'une plus grande quantité de cellules, la qualité inférieure des spermatozoïdes mobiles après congélation.

$$
\text { Reçu pour publication en juillet } 1965 .
$$

\title{
REMERCIEMENTS
}

Ce travail a pu être effectué grâce à un fonds de concours de l'U.N.C.E.I.A. et à l'aimable collaboration de l'administration et du personnel de la Coopérative d'Élevage et d'Insémination de la Loire (Montrond-les-Bains), organismes auquels je tiens à exprimer mes plus vifs remerciements

\section{SUMMARY}

\author{
EFFECTS OF MOTILA INSEMINATED SPERM NUMBER
}

ON THE FERTILIZING ABILITY OF FROZEN BULL SPERM

Sixteen ejaculates from 9 Charolais bulls were diluted in a skimmilk-fructose-glycerol extender and classicaly frozen by means of an alcool-bath and crushed dry-ice. Before ampulling, each ejaculate was divided into 3 portions of equal volume containing respectively 24,31 and 40 millions motile sperms per milliliter. The inseminations were done by four technicians, each of them receiving the same number of racks for each ejaculate and each treatment.

Of the overall number of 2602 first services, 866 were made with 24 million, 848 with $3 \mathrm{I}$ millions, 888 with 40 millions motile sperms before freezing. The corresponding non-return rate for 90-I 20 days were 6I.6-62.9 and 66.5 respectively. Only the difference between the 24 million and the 40 million lots was significant $(\mathrm{P}<0.05)$.

Because of the deep fall in the percentage of motile sperms during the freezing process, possibly owing to the fact that no selection on freezability had been preliminarily made, the 3 treatments expressed in terms of numbers of motile sperms after freezing, come down to I I.0; I 4.2 and I 8.3.

The trend indicated by the experiment agrees with the previous report of ERICKSON and GRAHAM (I959) and shows that the minimum sperm number required for an insemination is definitely higher when frozen semen is used as compared with fresh semen.

\section{RÉFÉRENCES BIBLIOGRAPHIQUES}

Emmens C. W., Martin I., I961. The effects of equilibration period and sugar content of the diluent on the survival and fertility of bull spermatozoa deep-frozen to $-79^{\circ} \mathrm{C}$. IVth Inter. Congr. animal.reprod artif. insem. The Hague.

Erickson W. F., Graham E. Fy, 1959. Factors affecting the fertility of frozen bovine spermatozoa. J. Dairy . Si., 42, 520-528. 
Foote R. H., DunN H. O., 1962. Motility and fertility of bull semen extended at high rates in yolk extender containing catalase. J. Dairy Sci., 45, $1237^{-1} 24 \mathrm{i}$.

Goffaux M., I964. Aptitude des taureaux utilisés en insémination artificielle à la production de semence capable de supporter la congélation. Ann. Zootech., 13, 87-92.

O'I Ell W. T., Almqutst J. O., 1958. Freezing bovine semen. IV. Fffect of freezing on the metabolic activity of bovine spermatozoa during and after storage at $-79^{\circ} \mathrm{C}$. J. Dairy Sci., 41, $179^{2-1} 799$.

Salisbury G. W., Van Demark N. L., 1961. Physiology of reproduction and artificial insemination of catle, 434-435. IV. H. Freemann and Co. San Francisco and London.

Sullivan J. J., Mixner J. P., I963. Effects of storage teniperature and length of storage time upon the post thawing motility and metabolic activity of frozen bull sperm. J. Dairy Sci., 46, 850-853. 